Session XXXX

\title{
Case study of the pedagogical impact of tablet PCs as a presentation medium in large-scale engineering classrooms
}

\author{
Sean P. Brophy ${ }^{1}$ and D. Greg D. Walker ${ }^{2}$ \\ ${ }^{1}$ Department of Biomedical Engineering, ${ }^{2}$ Department of Mechanical Engineering \\ Vanderbilt University, Nashville TN
}

\begin{abstract}
$\underline{\text { Abstract }}$
New information technologies promise to enhance engineering instruction by facilitating the learning process. A preliminary study examines the use of a tablet PC as a presentation platform for problem-solving methodologies used in a fourth year Mechanical Engineering class of 50 students. Typical presentation methods used in largescale classroom settings can inhibit students' ability to follow their instructor's thought process because either the format is too small to be viewed effectively by the entire class as in the case of whiteboards, or too static to illustrate the problem-solving process as in the case of overhead projection of transparencies or prepared Powerpoint slides. Tablet PCs connected to large-screen projection systems offer one solution for presenting material in a large-scale format while maintaining a dynamic presentation. We evaluate the potential of this classroom technology by considering both an instructional point of view and a learning point of view. In terms of instruction, the pen based tablet PCs offer several inherent advantages such as natural inclusion of complex digital media (graphs and charts) along side active concept development (instructor annotations). In other words, the instructor can make their thinking visual by maintaining a real-time mixture of complex engineering concepts and expert annotations. In addition, the instructor 1) is mobile, 2) can face the class and 3) does not obstruct the view of the material as it is presented. These logistic features provide a more fluid and coherent lecture, which should promote information gathering and understanding of the material by a student. In terms of learning, a student's comprehension is presumably enhanced due to the synchronization of note taking with verbal descriptions of the material. To test this hypothesis, a survey and focus group were used to determine students' perspective on the effects of the Tablet $\mathrm{PC}$ on their comprehension and learning compared to other classroom experiences that did not utilize the new platform. Results of the pilot study suggest that students are more likely to pay attention during the lecture and recognize the more salient points of the presentation. However, students who indicate that their prior knowledge is not sufficient to understand the basic were neutral on the benefits for using multimedia on the Tablet PC which may indicate that more needs to be done to prepare students for lecture. Overall, the findings are encouraging, and more research needs to be done to assess
\end{abstract}


increases in students' learning with highly synchronized video and audio support compared with static presentation of knowledge and audio annotation.

\section{$\underline{\text { Introduction }}$}

Many upper level undergraduate engineering courses have learning objectives leading to developing students' capable of solving complex computational problems involving mathematical relationships that define the principles of a domain. This requires developing the expertise to recognize how to mathematically describe a system's behavior. Therefore, a common instructional method is to derive fundamental equations that describe the behavior of a system and to demonstrate how an expert would approach the solution to multiple examples of how these equations apply to various contexts. This instructional method relies heavily on mathematical symbols as a descriptive language. Therefore, an instructor typically uses a white board to express their expert reasoning and explanation for how to approach and to solve problems related to that domain.

However, in a large lecture hall certain conditions make this less than optimal for all the students' learning. Alternative methods to the whiteboard could improve all students' classroom experience.

Many college classrooms now have the ability to project a computer display on a large screen for all students to view. Engineering instructors use this technology in a variety of ways. For example, instructors use videos to give students experience they may have never encountered before, such as, using a computational model to predict the behaviors of a turbine engine, or illustrate how various parameters of a system change using various visualization methods. Access to the web provides an additional set of resources that can be used in the classroom to increase students' ability to comprehend major topics presented during a class session. Some instructors use Powerpoint presentations to organize and structure their ideas, and resources, into an instructional lesson they will describe during classroom lecture and discussion. Engineering instructors can construct Powerpoint slides with mathematical expressions by transforming the equations into images using special editing tools. Then the instructor can use Powerpoint's animation features to emulate the derivation process previously conducted on the whiteboard. When students ask questions, the instructor can use the whiteboard to extend their explanation. These multimedia capabilities can provide engineering students a richer classroom experience because notes are well organized, the visual display is clear and unobstructed, and various multimedia formats can be used to assist in developing students' knowledge of the domain. Further, these notes can be posted in a "course management system" after class for students to review for homework and preparation for exams.

Portable Tablet Personal Computers, or simply Tablet PCs, can build and expand on these benefits of instructional technology in the classroom. The Tablet PC is a portable computer with a special pen that allows the user to write on the computer screen using natural handwriting, which displays as "digital ink" on the computer screen. The screen is similar to a standard laptop computer, but the total unit typically weighs much less than a standard laptop. The user can hold the device cradled in one arm, like a clipboard, and 
write with the other hand. Now an engineering instructor can face the class and converse with the students as he writes the notes on the Tablet PC, which is displayed on the large screen at the front of the class. The general process is directly analogous to the overhead projector and transparencies. However, several major differences make the Tablet PC more advantageous. First, the instructor does not need to stand in front of the screen and block students' view. Second, the instructors' hand and arm do not temporarily block the image while writing; therefore students have continuous flow of information as the instructor verbally describes the process. Third, the instructor can effortlessly switch between these hand notes to other resources on the Tablet PC's harddrive or through a wireless connection to the Internet.

The Tablet PC provides an interesting combination of traditional lecture style presentation and dynamic visual support provided by simulations and models, plus access to a wide range of other resources. We anticipate that this tool can enhance the classroom-learning environment by increasing an instructor's effectiveness for communicating information to his students by maintaining a clear, continuous presentation of lecture material. We expect students will find the Tablet PC easier to see and more expressive compared to standard whiteboard and Powerpoint presentations. Therefore, students will pay closer attention in class and could potentially increase their comprehension of the material during class.

In this study we investigated students' perception of this instructional method to improve visibility of the instructional materials, the expressiveness of the presentation and changes in their ability to learn in this classroom-learning environment.

\section{$\underline{\text { Method }}$}

This study evaluated students' perceptions of the benefits for using a Tablet PC as the major display medium in an engineering lecture compared to traditional use of a whiteboard and prepared Powerpoint presentations. The study was conducted in a fourth year Heat Transfer course in Mechanical Engineering. Forty-seven students were registered for this course that meets 2 times a week for one hour and fifteen minutes over a 13-week semester. A staggered baseline design was used to compare the two instructional methods.

In the first week of the course, the instructor conducted traditional lectures using prepared Powerpoint slides and the white board to derive equations and provided computational examples for solving heat transfer problems. The large lecture hall can accommodate 150 students and has sliding whiteboards at the front of the hall. Two smaller whiteboard are on either side of this the major whiteboard. The design of the room requires lowering a projection screen in front of the main whiteboards to display the Powerpoint presentation and images from a document camera. The primary source of lecture material was the standard whiteboard and lecture note combination. However, complex graphics or specific pages from the required text were often examined by lowering the projection screen to display computerized graphics or to display the document camera. Naturally, these alternate forms of display obscured the lecture existing lecture notes, and 
switching mediums generated momentary "downtime" when no additional information could be provided.

After the standard mode of operation using whiteboards, the instructor began using an NEC Versa LitePad slate-style Tablet PC as the central communication medium. His initial instructional method was to use the Tablet PC to present Powerpoint slides, which he annotated with the stylus. Blank slides were inserted into the PowerPoint presentation as "white" space to derive equations and demonstrate the problem solving process. The initial attempts to use this method were cumbersome because the Powerpoint program often froze while saving the annotations added during lecture. Ultimately, the instructor used Microsoft Journal Notes program standard with the XP operating system for the Tablet PC. Journal Notes provides the large screen space for presenting notes with minimal space dedicated to menus for program control. The instructor was able to easily switch pen colors to help emphasize key points and could quickly save a page and start a new page seamlessly. In addition, the instructor found it useful to select portions of the screen and relocate the text to make the presentation of the material even more structured. When a screen was filled, he would ask the class if they had any questions about that portion of the material, and if they were ready for him to continue to a clean "white" space. Note that the presentation format also allowed for display of material on a previous page above new space for new material (a scrolling mode). Students could request returning to any previous slides, which the instructor did willingly.

The instructor centralized all multimedia materials to the electronic presentation of materials to the large screen projection. In addition to using the Tablet PC as a whiteboard, the instructor often linked to web resources or prepared Labview virtual instruments, for example, to demonstrate pertinent concepts. Also, he used a document camera connected to the main projector to present static images from the book, and other sources, to share with the students. Therefore, the instructor could effortlessly move from one presentation medium to another with a few simple jesters with the Tablet PC stylus or the touch screen consol at the front podium.

\section{Instruments}

A student survey was constructed to measure students' perceptions of the contrast between both instructional methods. Items were constructed to measure the visibility of the materials, the clarity of expression by the professor, the ease of following a presentation, and improvements to their comprehension of the material. Several openended questions allowed students to share additional comments they had about the benefits and constraints of the Tablet PC in the classroom. Several of the items were worded in the negative tense to avoid biasing students toward a particular outcome related to our hypothesis. The items are listed in appendix A.

Half way through the semester a portion of a lecture period was used to administer the survey and conduct a focus group session led by the Director of the university's Center For Teaching. The instructor introduced the representative and stressed how important the results of this feedback were to him so he could improve the effectiveness of his 
teaching methods. He left the room and the students took approximately 15 minutes to complete the survey. Then the Director from the Center of Teaching led a 10 minute focus group when students could share their thoughts with the rest of the class and the Director could ask follow on questions to increase clarity of their responses. The first author of this paper also attended this session to take notes documenting students' comments. The surveys were anonymous, and the results were summarized and reported back to the instructor. Based on the preliminary results, the instructor made several modifications to the class structure and presentation based on the results.

\section{$\underline{\text { Results }}$}

41 students, of the 47 registered students, attended the class session when the survey was administered and all these students completed the survey. A frequency analysis of responses have been summarized and compared with the qualitative results from the survey to identify major trends in students' reaction to lecture based instruction using a Tablet PC as the central presentation medium. In addition, correlations were used to identify potential dependencies between instructional methods and students' perceptions of their learning in this course. Finally, the qualitative responses to the benefits and drawbacks to learning with the Tablet PC were coded into specific categories and a frequency analysis conducted on these comments.

The first 2 items in Figure 1 summarizes students' perception of the expressiveness and clarity of using the Tablet PC during lectures. The students agree that they like to watch the instructor develop equations and solutions to problems in real time on the Tablet PC better than from a Powerpoint presentation. Also they agree that it is easier to see the Tablet PC presentation compared to the whiteboard. One student commented - It's easy to read the class notes. It's easier for him to distinguish things using a different color font. I also prefer to see the problems solved in real time, as opposed to seeing a slide of the answer. (participant 21) The last items in Figure 1 summarize factors associated with students' comprehension of the materials presented with the Tablet PC and their general approach to how they process information during a class session. Most students either agreed, or were neutral, about using class time to make sense of the material as it is presented in class, rather than simply copying the notes to study later. Of these same students who agree, they tend to notice the salient concepts during class. In general, students do not find lectures harder to comprehend when presented on the Tablet PC versus a whiteboard and they are not overwhelmed with information when the Tablet PC is used in class. In fact, $44 \%$ of the students' agree that they pay more attention in class when the Tablet PC is used. One reason for this could be related to how the single display encourages students to attune to what the professor is saying rather than trying to catch up on note taking displayed on a different board. For example, several students made comments like this student -... it [tabletPC] allows me to focus on a single location rather than jumping around to all the different whiteboards. It also ensures the entire class is on the same concept because everyone must copy the notes from a slide before a new one can be started. Another student stated a similar idea as - [the tabletPC] forces us to keep up w/lecture and encourages you to not be late because the notes go to a new page after 1 is filled up (its not all up there like on the white board. 
These comments also highlight a different kind of interaction with the students that was not anticipated. We will discuss this more in the next section.

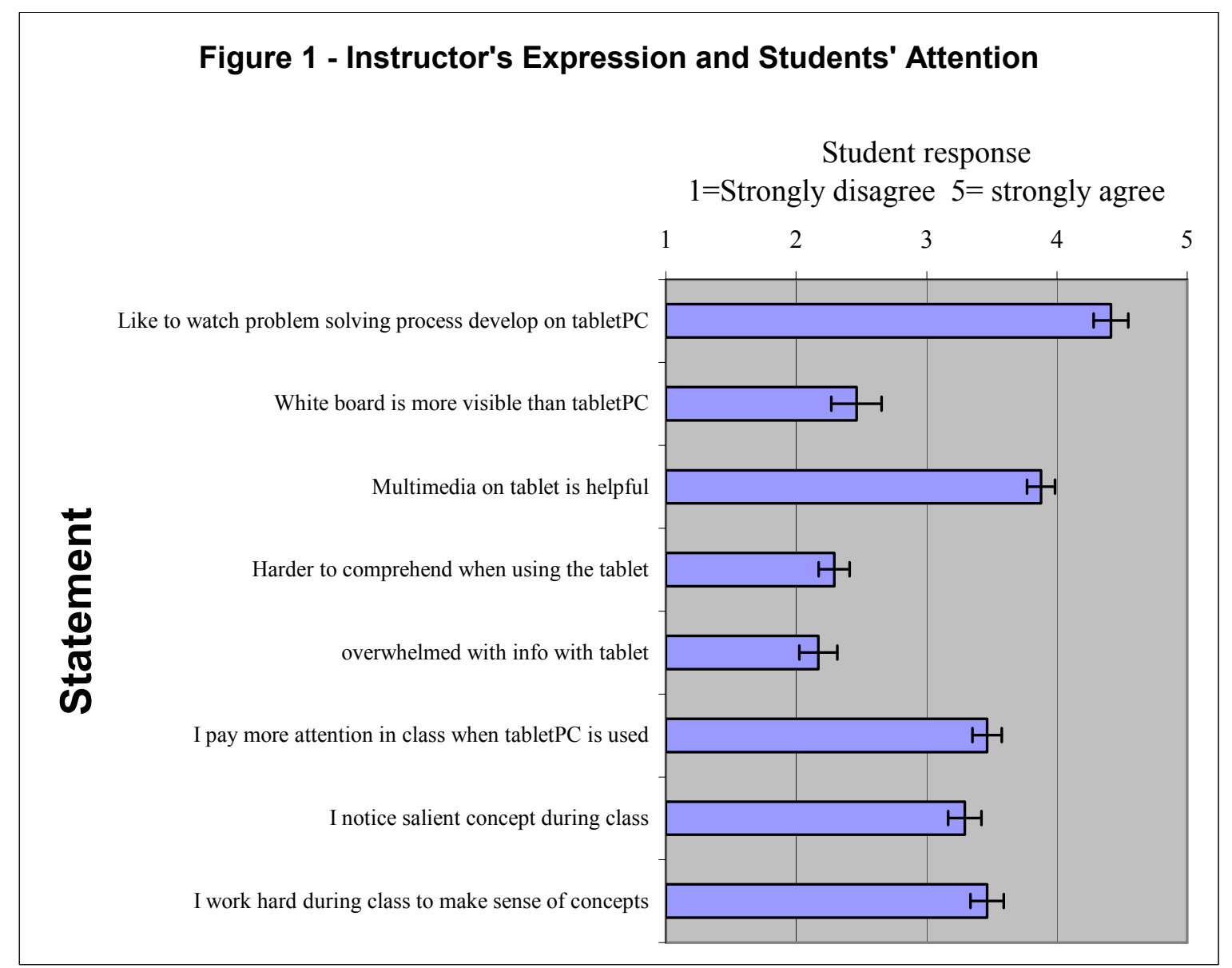

Figure 1 -

Figure 2 compares several items from Figure 1 with students' perceptions of how well their prior knowledge helps them understand the basics in the course and if they are often confused after lecture. Approximately $30 \%$ of the students were neutral on the benefits of the multimedia presentation. These exact same students also report not having sufficient knowledge to understand the basic concepts in this course. Three other students felt they have sufficient prior knowledge but agreed the multimedia presentations were helpful. The remainder of the class either agrees or is neutral when responding to how well their prior knowledge could be used to understand the basic concepts in this course.

Fifty six percent of the students agree that they are confused at the end of class. Half of these students were part of the group that identified not having sufficient knowledge to understand the basic concepts in this course. Much of this could be attributed to the complexity of the material for students to comprehend. Several students made comments similar to these one - tablet is a good way of presenting material however, the material itself is too hard to understand. Or -- make the course materials not so difficult. This 
might suggest that additional methods can be done to increase the potential for students to learn the materials in class.

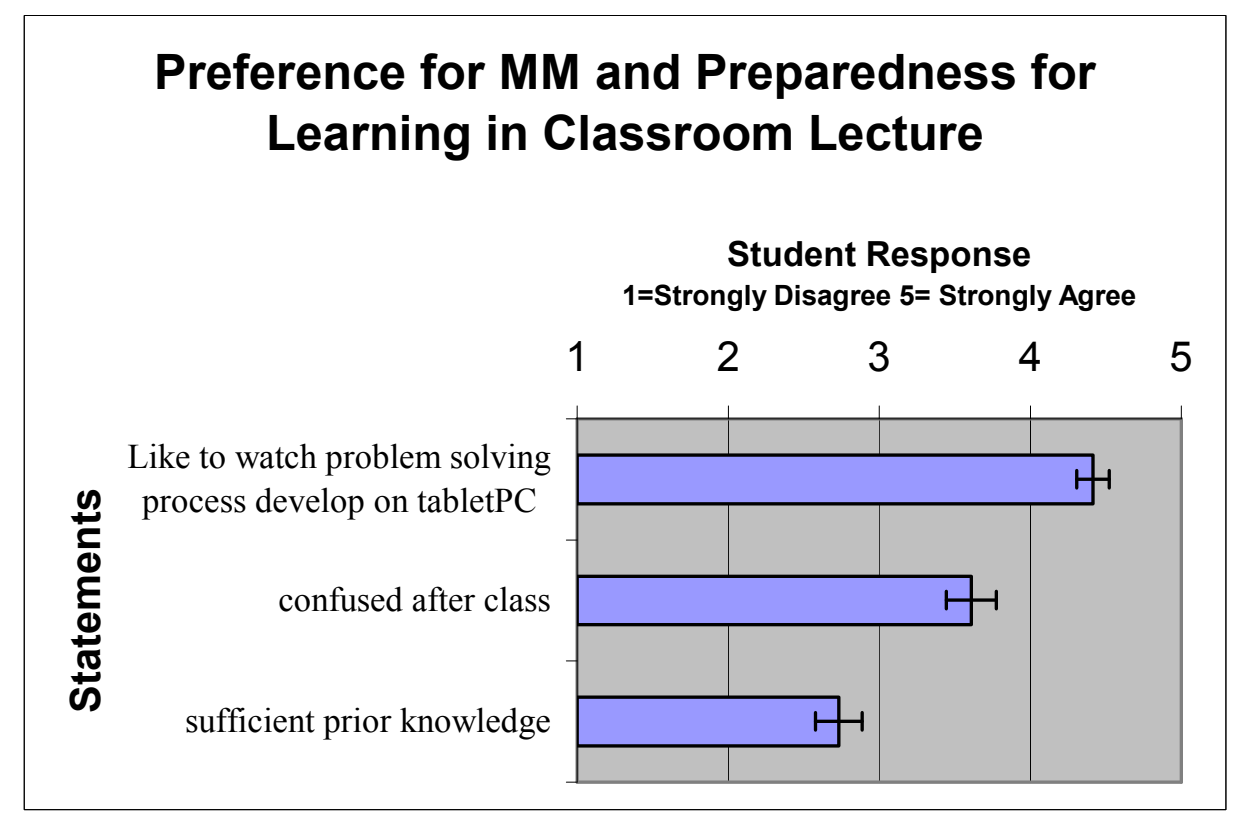

Figure 2 - Preference for Multimedia Presentation and Preparedness for learning in classroom lecture

The survey included two open-ended questions related to how the instructor's use of the Tablet PC has enhanced/inhibited ability to learn. Students provided a variety of responses to these questions. Table 1 provides a list of major categories of students' responses that emerged from the question on how the Tablet PC enhanced learning. Each student's response was placed into one or more category. For example, one student responded -

I love the idea. Incorporating different media (web, graphs, notes) all in one is great. Also he doesn't have to erase. When a page is full he simply uses a new one, then he can refer back to a previous one. I like the point about him being able to face us instead of turning around and blocking the board.

Each sentence in this response was summarized by one of these categories including a reference to using multimedia $(M M)$, efficient use of time because he doesn't have to erase the white board, faces the class increases interaction and finally more visible because he is not blocking the board. Every mention of one of these categories was tallied in the frequency count in Table 1. 
Table 1 - Categories of comments students made about professors use of Tablet PC for enhancing the ability to learn in the course.

\begin{tabular}{|l|l|l|}
\hline Categories & Description (examples) & Freq of comment \\
\hline visible & Easy to see, large font, no obstruction & 19 \\
\hline expression & $\begin{array}{l}\text { Easier for professor to explain, convenient for prof. , } \\
\text { dynamics of pres., spends more time on derivation, } \\
\text { ease of use }\end{array}$ & 10 \\
\hline history & Record of prior slides, can return for review & 10 \\
\hline face & $\begin{array}{l}\text { Facing the class while speaking, increases sense of } \\
\text { interaction }\end{array}$ & 6 \\
\hline MM & $\begin{array}{l}\text { Flipping between multimedia include document } \\
\text { projector }\end{array}$ & 6 \\
\hline attention & $\begin{array}{l}\text { Increases attention to material, encourage students to } \\
\text { view what instructor is talking about }\end{array}$ & 5 \\
\hline efficient & Saves time-don't have to erase & 4 \\
\hline
\end{tabular}

There were very few comments related to how the professor's use of the Tablet PC inhibited learning. Thirty percent of the students reported the pace was too fast. In general, the issues related to copying the notes generated by the professor before continuing to a new slide. However, they also acknowledged the professor's sensitivity to this issue because he asked if they had questions or were ready to continue before moving on to a blank screen. These same students also acknowledged that they valued the professors' ability to return to a previous slide to address questions. The only other comment was to use colors with sharp contrast to the white background (e.g. don't use yellow on white).

Finally, students were offered the opportunity to provide additional comments. The most frequent comment was to provide the lecture notes after class. Other students found the technology interesting, but wanted more help understanding these difficult concepts. Also, students found the technology very compelling and wanted to use their own pen based computer to capture lecture notes.

\section{$\underline{\text { Discussion }}$}

The professor's use of the Tablet PC clearly provided the anticipated benefits for assisting learning in a classroom setting. The visibility of the lecture notes was equal or better than the whiteboard for several reasons. The larger font and various colors made it easier for students to see the notes, even from the back of the room, compared to the whiteboard. In a large lecture hall it is difficult for students from all angles to see the whiteboard. Now the notes are displayed in almost three times the size of an original whiteboard, making it visible to all the students. In addition, the "digital ink" never runs dry whereas dry erase markers are notorious for going dry. Instructors can loose valuable class time searching for a pen. 
Students report an increase in their attention with the Tablet PC. These findings support the assumption that the unobtrusive view of the Tablet PC is an important contribution to the delivery of instruction, especially in an engineering classroom. The dynamic linking of visual and verbal information can have a large effect on a learner's ability to process and attune to information ${ }^{1}$. Dynamic animation of information is one instructional technique for helping people construct a mental model of how a system functions. ${ }^{2}$ Therefore, in theory the simultaneous construction of images (mathematical representations) with verbal descriptions could have an effect on students' ability to comprehend and attune to information in the classroom. However, what is not known is how well synchronized this dual information must be in order to assure students' processing of information. In the whiteboard, or overhead projector, presentation of materials, the professor typically blocks portions of the whiteboard while they are writing. His back is to the students, therefore, his verbal comments are made indirectly over their shoulder. Therefore, students must wait to see what he is talking about. This time delay to link the verbal description with the visual description may have an effect on students' comprehension of the material. Human's short term memory is limited ${ }^{3}$ which will put a larger burden on students' to process the information. Minimizing this delay could contribute to students ease for processing information. Additional research could be conducted to evaluate the interaction between comprehension and latency between presentation of the visual and verbal information.

Another factor influencing students' attention is narrowing the bandwidth of information delivery to a single screen. This can encourage students to keep pace with the instructor and process the information as the professor presents the information. Therefore, students may be more likely to spend their time processing the information as the instructor talks about it versus simply copying the information down in their notes and making sense of it after class. Most of these students do attempt to keep up with professor regardless of the delivery of information as indicated in the survey item in Figure 1.

Further, the dual presentation of visual and verbal information may be one reason why the majority of students prefer to watch the instructor develop and solve problems in real time on the Tablet PC rather than view materials from a prepared overhead slide projector. Additional explanations can be found in students' responses to items in the survey and their qualitative comments. For example, most of the students found the additional use of multiple media to be effective in helping them understand the concepts. Prior research related to media formats illustrates the potential of expressing ideas in multiple context and formats ${ }^{1}$ beneficial to students' comprehension.

We did not anticipate the students' positive reaction to having the professor facing them as he talked. From their comments, they identified a different connection with the professor as though they were engaged in a conversation. This could have an important affect on the classroom community that is formed, which invites students to either share information or ask questions. More research could be done to evaluate the benefits to students' attention and motivation to participate in the classroom lessons. In the future 
we could identify the kinds of question students ask to determine if they are engaging in the content.

The results of the survey highlight several opportunities for change to better meet the students' needs. Many students report the pace of the class was two fast. The increased efficiency of delivering the instructional materials could be one factor. No longer do students have transition breaks, "downtime", between media, or when an instructor must erase the whiteboard. Therefore, they do not have time to catch up on their notes, or to think more deeply about newly presented ideas. The instructor has been sensitive to this issue by stopping every so often to ask students if they have questions, or if they need additional time to complete their note taking. Other effective methods for encouraging reflection on the material is to ask thought provoking questions that require students to apply the new concepts and to solicit students' thoughts. Various classroom technologies like the Personal Response System ${ }^{4}$ can be used to poll student responses. The instructor can use this simple formative assessment method to evaluate if the majority of the class comprehends the material and if he should continue with the next topic of conversation, or continue with the current topic ${ }^{5,6}$.

Student preparation for class may be a limiting factor to the effectiveness of lecture based learning environments. Most every student favors the real time development of concepts; however, a significant portion of the students (30\%) report being neutral on the multimedia capabilities of the materials and report not having sufficient prior knowledge to understand the basic concepts. This might suggest that the time spent in the classroom providing background knowledge using multimedia may not be sufficient for all the students. What these students need is more preparation for class; then they can be told. Bransford and Schwartz ${ }^{7}$ emphasize this idea of constructing a "time for telling". They suggest that a lecture can be an excellent method of instruction when the learners prepare to be told the information. However, if students do not have a sufficient experience to comprehend what, when and how the new knowledge is applied, then they are less likely to understand the utility of the formal, more abstract, representations of the knowledge. Therefore, increasing students' comprehension of the material and reducing how many students leave the class confused, we could consider using short pre-class activities. These pre-class activities can engage students in activities that require them to apply what they know to solve a challenge and to identify what more they need to now to solve the problem. These should help them generate questions they need answered. If designed well, the lecture will provide the answers to these questions. Now the students are primed for the lecture, which will increase the likelihood that everyone will comprehend the major principles of the lecture and be better prepared to apply this knowledge to homework.

\section{Summary}

This initial study evaluated students' perception of how well the instructor can express his ideas using a Tablet PC. We found that students identified similar benefits for the technology as we did, and identified several other benefits to learning. For example, students do value the clarity of the presentation and the demonstration of the domain 
expertise. The found the multimedia presentation helpful and report a higher attention rate when the TabletPC is used. Their responses provide hints that our rational for the inclusion of this technology to enhance comprehension and motivation to attune is correct. Additional studies need to be conducted to measure the cognitive benefits of this technology on students' ability to learn in this type of lecture-based course that relies heavily on mathematical representations. Also, additional activities before class my increase students' ability to comprehend the lecture better resulting in fewer students leaving class confused by the fundamental concepts.

\section{Acknowledgements}

This work was supported by the Engineering Research Centers Program of the National Science Foundation under Award Number EEC9876363. Also, the office of the Provost provided funding for the classroom technology under the Innovation in Teaching and Learning with Technology Program.

\footnotetext{
References

${ }^{1}$ Mayer, R. E., \& Moreno, R. (1998). A split-attention effect in multimedia learning: Evidence for dual processing systems in working memory. Journal of Educational Psychology, 90(2), 312-320.

${ }^{2}$ Hegarty, M. (2004) Dynamic visualizations and learning: getting to the difficult questions. Learning and Instruction, 14(3) pp 343-351.

${ }^{3}$ Miller, George A. "The Magical Number Seven Plus or Minus Two: Some Limits on our Capacity for Processing Information." The Psychological Review, 63 (1956): 84-97.

${ }^{4}$ Interwrite Personal Response System - http://www.gtcocalcomp.com/interwriteprs.htm

${ }^{5}$ Roselli, R.J. and Brophy, S.P. (2002) Exploring an electronic polling system for the assessment of student progress in two biomedical engineering courses. Proceedings of the American Society for Engineering Education (CD-ROM DEStech Publications) Session 2609: 11 pages.

${ }^{6}$ Roschelle, J., Penuel, W. R., \& Abrahamson, L. A. (2004). The networked classroom. Educational Leadership, 61(5), 50-54.

7 Schwartz, D. L. and Bransford, J. D.(1998) A time for telling," Cognition andInstruction, 16 (4), pp.475522.
}

\section{Biographies}

SEAN P. BROPHY - Brophy received his B.S. degree in Mechanical Engineering from the University of Michigan, an MS in Computer Science from DePaul University and PhD in Education and Human Development from Vanderbilt University. He currently is an Assistant Research Professor in the Department of Biomedical Engineering at Vanderbilt and co leader of the Learning Sciences thrust. His current research interests relate to using simulations and models to facilitate students' understanding of difficult concepts within engineering as part of the VaNTH Engineering Research Center (ERC). 
D. GREG WALKER - Walker received his M.S. and B.S. in Mechanical Engineering from Auburn University and his Ph.D. in Mechanical Engineering from Virginia Tech. He is currently an Assistant Professor in the department of Mechanical Engineering at Vanderbilt University. His interest in teaching has prompted him to initiate the program of studying the pedagogical effects of using Tablet PCs in largescale classrooms. 